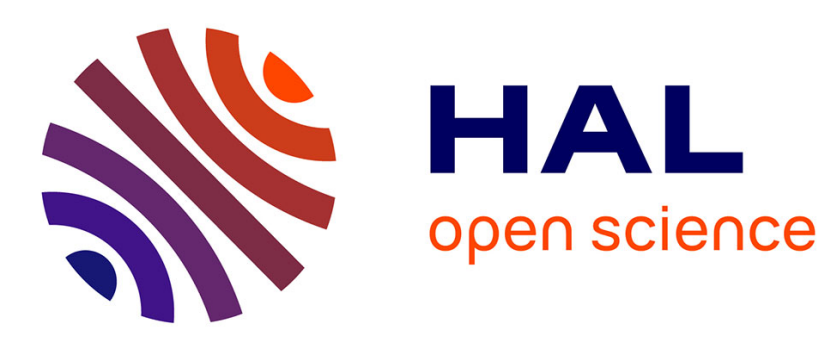

\title{
La place et le rôle des femmes dans la politique étrangère de la France contemporaine
}

\author{
Yves Denéchère
}

\section{To cite this version:}

Yves Denéchère. La place et le rôle des femmes dans la politique étrangère de la France contemporaine.

Vingtième siècle. Revue d'histoire, 2003, pp.89-99. halshs-01089571

\section{HAL Id: halshs-01089571 \\ https://shs.hal.science/halshs-01089571}

Submitted on 2 Dec 2014

HAL is a multi-disciplinary open access archive for the deposit and dissemination of scientific research documents, whether they are published or not. The documents may come from teaching and research institutions in France or abroad, or from public or private research centers.
L'archive ouverte pluridisciplinaire HAL, est destinée au dépôt et à la diffusion de documents scientifiques de niveau recherche, publiés ou non, émanant des établissements d'enseignement et de recherche français ou étrangers, des laboratoires publics ou privés. 


\section{La place et le rôle des femmes dans la politique étrangère de la France contemporaine}

\section{Un regard sur l'historiographie}

La place et le rôle des femmes dans la politique étrangère de la France n'ont jusqu'à maintenant pas été étudiés. Pourtant, on peut penser qu'au moins deux champs de la recherche historique devraient s'y intéresser : l'histoire des relations internationales et l'histoire des femmes. Pierre Renouvin et Jean-Baptiste Duroselle, les grands historiens qui ont défini l'histoire des relations internationales, insistent sur l'importance des « forces profondes » et le rôle de «l'homme d'Etat» face à ces forces. Mais ils ne parlent des femmes que lorsqu'ils évoquent le rôle que les épouses et les maîtresses peuvent jouer auprès des décideurs ${ }^{1}$. Il est vrai que lorsqu'ils écrivent leur Introduction à l'histoire des relations internationales, en 1964, bien peu de femmes occupent de par le monde des postes à responsabilité politiques et/ou diplomatiques de premier plan. Par la suite de nombreux travaux ont été entrepris et ont permis de mieux connaître toutes les composantes des forces profondes et toutes les facettes des hommes d'Etat. Mais les femmes sont demeurées dans l'oubli, même si l'on doit signaler le récent numéro de Guerres Mondiales et Conflits Contemporains, intitulé : Les femmes et la guerre $^{2}$. Pourtant, les années passant, elles accédaient, certes lentement mais indéniablement, à des responsabilités politiques et diplomatiques qui leur ouvraient modestement le domaine des relations internationales.

Du côté de l'histoire des femmes, où tout était à faire dans les années 1970, les chercheuses - car les chercheurs étaient et restent peu nombreux dans ce domaine - devaient parer au plus pressé. Michelle Perrot et d'autres définissaient alors les grands chantiers les plus urgents à mettre en oeuvre ${ }^{3}$. On comprend que la diplomatie, qui apparaissait comme un territoire exclusivement réservé aux hommes, pouvait attendre et passer après l'histoire des mentalités, du travail des femmes, de la question des femmes en politique, des mouvements de femmes et du féminisme... Cependant, dans le cadre d'études consacrées aux premières femmes accédant à certains diplômes ou à certaines professions, on pouvait s'attendre à l'évocation de Suzanne Borel. Encouragée par André Siegfried, elle fut la première lauréate du «grand concours» d'entrée au Quai d'Orsay en 1930. Elle joua un grand rôle au lendemain de la Deuxième Guerre mondiale auprès de son mari, Georges Bidault. Mais dans ses ouvrages de souvenirs, qu'elle signe d'ailleurs Suzanne Bidault ${ }^{4}$, la «première » rejette tout féminisme et stigmatise les effets pervers de ce mouvement. De plus, le fait qu'elle suive son mari dans l'exil après ses prises de position pro-OAS, n'a sans doute pas incité à s'intéresser à son cas. Aussi, on ne trouve que quelques lignes, au mieux quelques pages, consacrées à Suzanne Borel dans des ouvrages généraux sur l'histoire des femmes ${ }^{5}$. Heureusement, tout récemment, des travaux universitaires se sont attachés à la mieux

\footnotetext{
${ }^{1}$ Pierre RENOUVIN et Jean-Baptiste DUROSELLE, Introduction à l'histoire des relations internationales, Paris, Armand Colin, 1964, p. 379 (4 ${ }^{\mathrm{eme}}$ édition, 1991).

${ }^{2}$ Guerres Mondiales et Conflits Contemporains, $\mathrm{n}^{\circ} 198$, juin 2000.

${ }^{3}$ Voir Michelle PERROT, Les femmes ou les silences de l'Histoire, Paris, Flammarion, 1998, notamment l'introduction générale, rappelant les différentes étapes de la construction de l'histoire des femmes.

${ }^{4}$ Suzanne BIDAULT, Par une porte entrebâillée ou comment les Françaises entrèrent dans la Carrière et Je n'ai pas oublié, Paris, La Table Ronde, 1971 et 1972.

${ }^{5}$ Par exemple dans: Marie-Thérèse GUICHARD, Les égéries de la République, Paris, Payot, 1991, pages 242244 ; William GUERAICHE, Les femmes et la République, Paris, Editions de l'Atelier/Editions ouvrières, 1999, p.54-55.
} 
connaître $^{6}$, mais il n'existe toujours pas de thèse sur ce personnage. La « première » ayant peu retenue l'attention, les suivantes furent d'autant plus facilement oubliées.

De nombreux ouvrages présentent à juste titre l'ouverture aux femmes du concours d'entrée à l'ENA comme une avancée significative dans le combat pour l'égalité des sexes et l'accession aux responsabilités dans 1 'administration française ${ }^{7}$. Mais bien peu se préoccupent des effets de cette nouveauté dans la politique étrangère de la France. Et pour cause ! répondra t-on, puisqu'il y en eut si peu. Il n'y a guère que des livres destinés au grand public et se présentant comme des chronologies très générales, pour signaler que la première femme ambassadeur fut nommée en 1972 seulement. Tout ce que l'on apprend, c'est que mademoiselle Marcelle Campana est alors chargée de représenter la France au Panama, après avoir été nommée consul en $1967^{8}$. Mais de son parcours et ses engagements personnels, sur les conditions de sa nomination, sur les réactions du monde politique et diplomatique et de l'opinion publique on ne sait rien.

Pour compléter ce tableau peu encourageant, ajoutons que l'histoire des relations internationales et l'histoire des femmes sont des domaines qui s'ignorent le plus souvent, chacun ayant quelque chose à reprocher à l'autre, et pas toujours sans raison. Les uns se méfient d'une histoire militante, quand les autres constatent qu'on fait une « histoire sans les femmes ${ }^{9}$. Les rares travaux croisant les deux disciplines, notamment ceux de Françoise Gaspard, portent essentiellement sur les questions du genre et sur l'implication des mouvements de femmes dans les relations internationales d'aujourd'hui ${ }^{10}$. Ailleurs dans le monde, et toujours autour de la question du genre, il faut noter un article pionnier de Rosemary Foot qui en 1990 se demandait où sont les femmes dans l'étude des relations internationales ${ }^{11}$. Au Canada, Madame Claire Turenne Sjolander s'intéresse également à ces questions et notamment à la manière dont les féministes canadiennes analysent la politique étrangère de leur pays ${ }^{12}$. Un recensement international complet des travaux effectués ou en cours dans ce domaine permettrait sans doute d'orienter les recherches à mener en France.

Ces quelques remarques, qui volontairement posent plus de questions qu'elles apportent de réponses, cherchent seulement à pointer quelques raisons qui expliquent que la place des femmes dans les relations internationales en général et dans la politique étrangère de la France en particulier demeure dans l'ombre de l'histoire. Pourtant, les domaines à explorer

\footnotetext{
${ }^{6}$ On peut signaler l'intervention de Michel MARBEAU, « Une timide irruption des femmes dans la politique étrangère de l'entre-deux-guerres », lors de la journée d'études du 21 septembre 2002 consacrée aux Femmes dans la politique étrangère de la France au XXème siècle : études et témoignages, Université d'Angers. En 2002, sous la direction de Robert FRANK, Elodie LEJEUNE a réalisé un mémoire de maîtrise sur Suzanne Borel intitulé : Suzanne Bidault, une pionnière oubliée.

${ }^{7}$ Michel DEBRÉ, Trois Républiques pour une France, Paris, Albin Michel, 1984, tome I p.373-374. Guy THUILLIER, Les femmes dans l'administration française depuis 1900, Paris, Presses Universitaires de France, 1988.

${ }^{8}$ La «première » n'est pas mademoiselle Dienesch nommée au Luxembourg en 1972 comme l'indique Guy Thuillier dans Les femmes dans l'administration... op. cit., page 92. Mademoiselle Marie-Madeleine Dienesch, ancien secrétaire d'Etat (1968-1972), fut bien nommée ambassadeur au Luxembourg mais en 1975, soit trois ans après la nomination de Marcelle Campana.

${ }^{9}$ Pour reprendre le titre du colloque de Rouen de 1997 : L'histoire sans les femmes est-elle possible ?, AnneMarie SOHN et Françoise THELAMON (dir.), Paris, Perrin, 1998.

10 Notamment, Françoise GASPARD, «les femmes dans les relations internationales », dans Politique étrangère, $\mathrm{n}^{\circ} 3-4,2000$, pages 731-741; «les "droits de la femme" : construction d'un enjeu en relations internationales », La Revue Internationale et Stratégique, n 47 , automne 2002, pages 46-52.

${ }^{11}$ Rosemary FOOT, «Where are the women? The gender dimension in the study of international relations », in Diplomatic History, n4, vol. 14, 1990.

12 Claire TURENNE-SJOLANDER, Deborah STIENSTRA et Heather SMITH, Discours sexospécifiques, pratiques sexospécifiques, la politique étrangère canadienne revisitée par les Féministes, table ronde du CCDPE (Centre Canadien pour le Développement de la Politique Etrangère), Winnipeg et Québec, mai et juin 2000.
} 
sont nombreux et nous proposons d'en évoquer quelques-uns ici avant de définir une méthode de travail.

\section{Les femmes et la prise de décision en politique étrangère}

Les décisions de politique étrangère sont, par essence, de nature politique. La question qui se trouve posée est de savoir si les femmes, dans les postes à responsabilité politique qu'elles ont occupés, ont pris part à la prise de décision en politique étrangère. L'échelon le plus élevé en la matière est la Présidence de la République qui n'a jamais été exercée par une femme. Cependant, on peut se demander quels rôles ont joué les femmes au sein de la «cellule diplomatique » de l'Elysée. L'autre pôle de l'exécutif, c'est-à-dire Matignon, ne s'occupe pour ainsi dire de diplomatie que lors des périodes de cohabitation politique. Rappelons qu'il n'a été occupé qu'une fois par une femme, madame Edith Cresson, en 19911992, et pas pendant une période de cohabitation. Plus intéressant sans doute est l'étude de ce qui se passe au Quai d'Orsay. Il n'y a jamais eu de femme ministre des Affaires étrangères en France. C'est l'un des rares portefeuilles à avoir été constamment réservés aux hommes avec ceux de l'Intérieur, des Finances... Le bastion de la Défense étant tombé en 2002. Cependant, des femmes ont pu participer à la prise de décision au sein du cabinet du ministre, mais pour le dire, il faudrait d'abord étudier leurs fonctions et leur influence. Si le ministère a toujours échappé aux femmes, pour la première fois en 1984, une femme, madame Catherine Lalumière, devenait secrétaire d'Etat auprès du ministre des Relations extérieures. Puis en 1988, madame Edwige Avice était nommée ministre délégué auprès du ministre des Affaires étrangères. En tout, six femmes ont exercé les fonctions de secrétaire d'Etat ou de ministre délégué. A la moitié d'entre elles, on a souvent confié des attributions qu'il faut bien qualifier de secondaires, comme la francophonie - pour deux d'entre elles - une autre se voyant confier l'action humanitaire et les droits de l'homme ${ }^{13}$.

Les Affaires européennes sont également plus volontiers confiées à des femmes. En effet de 1984 à 1986 et de 1988 à 1993, un secrétariat d'Etat puis un ministère indépendant et un ministère délégué en charge de ces questions sont confiés à des femmes ${ }^{14}$. Les raisons de cette particularité restent à définir mais on peut avancer sans risque que dans les années 1980 ces postes n'étaient pas les plus recherchés par la classe politique masculine. On retrouve d'ailleurs la même relative "sur-représentation féminine » française au Parlement européen, dont la présidence est assurée par deux fois par des Françaises : mesdames Simone Veil et Nicole Fontaine, et au sein de la commission européenne de Bruxelles avec mesdames Scrivener et Cresson. Il est notoire que les hommes politiques français ont toujours privilégié l'engagement politique national et il semble qu'ils abandonnent aux femmes les responsabilités et les mandats européens qui leur apparaissent secondaires. Dans les postes qu'elles occupent dans les institutions européennes ou françaises, de quelle marge de manœuvre les femmes disposent-elles exactement en matière de politique européenne ? Estelle différente, plus large, de celles dont elles disposent dans d'autres domaines ${ }^{15}$ L'interaction entre le politique et la place des femmes dans la politique étrangère de la France est également posée par le très petit nombre de femmes députés ou sénateurs. Ce qui implique

\footnotetext{
${ }^{13}$ Francophonie : mesdames Catherine Tasca (1991-1993) et Margie Sudre (1995-1997); action humanitaire et droits de l'homme : madame Lucette Michaux-Chevry (1993-1995).

${ }^{14}$ Secrétaire d'Etat: Madame Catherine Lalumière (1984-1986); ministre des Affaires européennes : madame Edith Cresson (1988-1990) ; ministre délégué chargé des Affaires européennes : madame Elisabeth Guigou (1990-1993) : madame Noëlle Lenoir nommée en 2002.

${ }^{15}$ Voir Françoise GASPARD (dir.), Les femmes dans la prise de décision en France et en Europe, Paris, L'Harmattan, 1997.
} 
ipso facto leur faible représentation au sein des deux commissions des Affaires étrangères du parlement.

En mai 2001, Monsieur Loïc Hennekine, secrétaire général du Quai d'Orsay, affirmait : "le Ministère est très conscient de la place des femmes dans notre politique étrangère et il s'emploie à renforcer celle-ci en confiant à des femmes des postes à responsabilité au sein de notre diplomatie ${ }^{16}$. Voyons de plus près ce que cela veut dire.

\section{La féminisation du ministère des Affaires étrangères}

Les premières femmes à entrer au Quai d'Orsay avant 1914 furent quelques «dames sténodactylographes ». Puis, suivant une évolution générale à tous les ministères à la fin des années 1920, les Affaires étrangères ouvrent aux femmes des emplois de "rédacteurstraducteurs » et de secrétaires administratives, mais en nombre limité à quelques unités ${ }^{17}$. L'apparition de ces premières femmes dans les bureaux du Quai d'Orsay provoque d'ailleurs des réactions assez inégales parmi les habitués des lieux. Jean Giraudoux note en 1934 que «bien rare est le chef français qui élimine entre sa secrétaire et lui tout rapport personnel. Dans son bureau, le chef français est comme le mari français dans son ménage (...) Partout ou il passe (...) le Français recrée le couple. C'est très gentil de sa part, mais l'initiative et l'individualité féminine n'en sont que difficilement accrues ${ }^{18}$. Pour sa part, Gheusi évoque « les femmes qui dévorent la vie des maîtres de l'heure », et en particulier « la dactylo [qui] si elle est jolie, ambitieuse et libre joue un rôle excessif auprès de son patron ». Et d'affirmer que «celle de Philippe Berthelot eut raison d'un organisme exceptionnel ${ }^{19}$. En 1928, un décret permet aux femmes de se présenter au concours d'admission dans les carrières diplomatiques et consulaires sous la pression d'associations féministes et de diverses personnalités dont Louis Marin. C'est d'ailleurs sa pupille, Luce Camuzet, qui est la première femme candidate... mais elle ne sera pas admise $!^{20}$

En réussissant, elle, le concours en 1930, Suzanne Borel ouvre une nouvelle porte permettant l'entrée des femmes au Quai d'Orsay, mais ne peut guère aller plus loin. Giraudoux a raconté comment et pourquoi Suzanne Borel («Mlle X... » sous sa plume) se vit interdire les postes extérieurs ${ }^{21}$. Après la guerre, elle dirige le cabinet du ministre des Affaires étrangères Georges Bidault (septembre 1944-décembre 1946). Après leur mariage, et ne voulant pas jouer le double rôle d'épouse et de conseillère - du moins officielle - elle se retire provisoirement de l'administration. L'ouverture des postes extérieurs aux femmes en 1945, puisqu'elles ont enfin obtenu les droits civiques qui leur permettent d'assurer les fonctions d'officiers d'état civil, ne règle pas tout, loin de là. Bien qu'elle soit la première femme devenue ministre plénipotentiaire, Suzanne Borel ne sera jamais ambassadrice... sinon en tant qu'épouse de Georges Bidault. Le parcours de Suzanne Borel révèle bien des résistances qui demeurent après la Seconde Guerre mondiale. Le venin distillé par Roger Peyrefitte à l'égard

\footnotetext{
${ }^{16}$ Lettre de Son Excellence Monsieur Loïc Hennekine à l'auteur, 23 mai 2001.

17 Guy THUILLIER, Les femmes dans l'administration... op.cit., Cet ouvrage demeure le meilleur pour appréhender, par bribes, l'évolution de la place des femmes dans le ministère des Affaires étrangères jusque dans les années 1970, notamment p. 28 et 86.

${ }^{18}$ Jean GIRAUDOUX, La Française et la France, Paris, Gallimard, 1951, (texte d'une conférence intitulée « la femme devant l'univers » prononcée en 1934), p. 204-205.

${ }^{19}$ GHEUSI, Cinquante ans de Paris, paris, 1941, tome II : Leurs femmes, p. 346. Philippe Berthelot fut secrétaire général du ministère des Affaires étrangères de 1920 à 1922 et de 1925 à 1933.

${ }^{20}$ Guy THUILLIER, Les femmes dans l'administration ... op.cit., p.53-54.

${ }^{21}$ Jean GIRAUDOUX, La Française et la France...op.cit., p. 194-197.
} 
de «mademoiselle Crapote », est à cet égard très expressif ${ }^{22}$. Interdites de fait de postes à l'étranger et de perspectives de carrières satisfaisantes, les jeunes femmes sortant de l'ENA hésitent à entrer dans la diplomatie. En attendant, le Quai d'Orsay féminise son administration, même s'il s'agit d'un mouvement qui affecte surtout les grades les plus bas de l'échelle administrative. De nombreuses études dans différents secteurs d'activité ont bien montré que l'accès des femmes à certains métiers ou postes ne signifie pas l'égalité devant les carrières.

Il faut bien faire la distinction entre les grades et les emplois. A la fin des années 1970, $32 \%$ des postes du ministère des Affaires étrangères sont occupés par des femmes. Si 30,6 \% des personnels de catégorie B sont des femmes, elles ne constituent que 9,1\% des personnels de catégories A (il faut noter que les moyennes nationales de l'administration sont respectivement de 58 et $31 \%$ ). Ainsi, $25 \%$ des secrétaires de chancelleries sont des femmes, mais seulement $5 \%$ des secrétaires et des conseillers des Affaires étrangères et $0,5 \%$ des ministres plénipotentiaires. Bien entendu, serait-on tenté d'ajouter, aucune femme n'avait été élevée à la dignité d'ambassadeur de France. Sans trop multiplier des chiffres qu'il conviendrait d'analyser précisément, on peut ajouter que $58 \%$ des secrétaires administratives et $57 \%$ des traducteurs sont des femmes ${ }^{23}$. Ces chiffres n'étonnent guère, ils reflètent un phénomène bien connu qui est celui de la féminisation des postes subalternes. Plus remarquable est le fait que le cadre d'Orient apparaît plus féminisé que le cadre général. Sans doute en raison des compétences linguistiques très pointues qui sont exigées et du faible nombre de candidats. En ce qui concerne les emplois, seulement trois femmes ont alors exercé des responsabilités de chefs de mission (mesdemoiselles Campana, Dienesch et Malitchenko). A l'administration centrale, quelques postes de sous-directeurs, qui restent à recenser avec précision, sont occupés par des femmes, mais encore aucun poste de directeur. Les raisons invoquées pour justifier cette situation sont bien connues: vie familiale difficilement conciliable avec des postes à l'étranger, affectations de femmes «délicates sinon impossibles » dans les pays où « la condition féminine est inférieure », mais aussi conscience des jeunes femmes qui sortent de l'ENA que leur carrière sera plus facile à construire dans d'autres ministères qu'au Quai d'Orsay... Jean Baillou concluait que le nombre important de femmes situées au bas de l'échelle devait inéluctablement profiter des promotions à venir, et il invitait le Quai d'Orsay à agir en ce sens ${ }^{24}$. Plus de vingt ans plus tard, où en est-on ?

Il y a eu une incontestable évolution dans le sens d'une plus grande féminisation du personnel du ministère des Affaires étrangères. Désormais, les femmes occupent $18 \%$ des postes de conseillers des Affaires étrangères et $34 \%$ des postes de secrétaires principaux et secrétaires des Affaires étrangères ${ }^{25}$. Le cadre d'Orient est toujours plus féminisé que le cadre général. $6 \%$ des ministres plénipotentiaires de deuxième et première classes sont des femmes, mais une seule femme a accédé au grade de ministre plénipotentiaire hors classe. A ce jour, il n'y a toujours pas de femme parmi les ambassadeurs dignitaires (on en compte 4 en exercice et une cinquantaine à la retraite). Le constat d'une féminisation en nombre et en qualité est donc fait, mais elle ne touche pas encore les plus hauts barreaux de l'échelle ${ }^{26}$. Est-ce seulement un effet du retard pris antérieurement et qui s'explique par l'avancement normal des carrières ou peut-on trouver d'autres explications ? Y-a t-il des abandons de carrières par

\footnotetext{
${ }^{22}$ Roger PEYREFITTE, La fin des ambassades, Paris, Flammarion,1953. Dans ce roman autobiographique qui le met en scène sous le nom de «Georges », c'est Suzanne Borel qu'il faut reconnaître derrière le personnage de « mademoiselle Crapote ».

${ }^{23}$ Jean BAILLOU, Les Affaires étrangères et le corps diplomatique français, tome II : 1870-1980, Paris, CNRS, 1984, p. 910.

${ }^{24}$ Jean BAILLOU, Les Affaires étrangères... op.cit., p. 911

${ }^{25}$ Calculs réalisés à partir des données de l'Annuaire Diplomatique et consulaire, édition 2000.

${ }^{26}$ Ce constat est fait par Eva DALAK, La place des femmes au Quai d'Orsay, mémoire de DEA de Sciences politiques, Université Paris I, 1998, inédit, 135 pages.
} 
les femmes, et si oui pour quelles raisons ? L'évolution des carrières des femmes et de celles des hommes sont-elles parallèles et identiques ? Voilà encore un champ de recherche qui devra être labouré afin d'apporter des réponses claires et de supprimer tous les phantasmes et les stéréotypes imaginables. Une certitude demeure néanmoins, c'est que les femmes sont toujours plus nombreuses en proportion au bas de l'échelle et cantonnées dans des affectations que certains archaïsmes considèrent comme féminines. Ainsi, $66 \%$ des interprètes et $80 \%$ des traducteurs sont des femmes. Les huit fonctionnaires chargées du Service social sont toutes des femmes.

En ce qui concerne les emplois, les ambassadrices vont retenir ici notre attention, mais les postes à responsabilité dans l'administration centrale du Quai d'Orsay méritent également une attention particulière. En 1986, madame Isabelle Renouard devient la première femme nommée à un poste de directeur au Quai d'Orsay, «apparemment le plus misogyne des ministères avec l'Intérieur », «conformément à des traditions fort anciennes » ${ }^{27}$. Depuis, une douzaine de femmes ont occupé des postes de ce type. Là encore, il faut déterminer quelles ont été leurs actions, leur influence, leur place dans la hiérarchie du Quai d'Orsay, leurs relations avec les personnels dont elles étaient responsables, etc..

\section{Le cas particulier des ambassadrices}

La nomination de femmes ambassadeurs à partir des années 1970 pose une multitude de questions et nécessite des adaptations de la diplomatie française jusque dans ses aspects les plus protocolaires ${ }^{28}$. On peut commencer par évoquer la féminisation des mots. Jusqu'à une période très récente, on a uniquement utilisé le terme «ambassadeur » et les femmes chefs de mission à l'étranger sont donc appelées « Madame l'ambassadeur ». Dans l'édition 2002 de l'Annuaire Diplomatique, dans la liste chronologique des ambassadeurs, les femmes sont toutes appelées ambassadrices. Dans les éditions précédentes on réservait ce terme aux femmes nommées après 1998, année d'une circulaire du Premier ministre Lionel Jospin, portant sur «la féminisation des noms de métier, fonction, grade ou titre » en rappelle une autre datant de 1986 et jamais appliquée ${ }^{29}$. Elle concerne entre autres les diplomates. Désormais, les termes « ambassadrice » et « consule » sont utilisés par l'administration. Cette mesure est d'ailleurs diversement appréciée par les ambassadrices elles-mêmes. Une ambassadrice à la retraite écrit même : "nommée bien avant 1998, pour moi, l'ambassadrice reste l'épouse de l'ambassadeur et je ne reconnais à aucun ministre, fut-il le premier d'entre eux, de légiférer sur la langue française ». Et d'évoquer Madame Hélène Carrère d'Encausse, qui à l'Académie française «s'abstient de ces futiles et si féminins enfantillages». La conclusion est nette : «ambassadeur je fus, ambassadeur je reste ${ }^{30}$. Les courriers que les ambassadrices envoient aujourd'hui comportent souvent encore l'en-tête «l'Ambassadeur ». De même, des sites Internet d'ambassades, dans leur présentation générique, proposent le "portrait de l'Ambassadeur » ou le «mot de l'Ambassadeur », même lorsqu'il s'agit d'une femme. En revanche, d'autres diplomates revendiquent et utilisent le terme « ambassadrice », et expriment leur souci d'une plus grande représentation des femmes parmi les chefs de mission français à l'étranger ${ }^{31}$.

\footnotetext{
${ }^{27}$ Guy THUILLIER, Les femmes dans l'administration... op.cit., p. 86 et 93.

${ }^{28}$ Nous avons traité de cette question lors du colloque Le genre des territoires, Le Plessis-Macé, mai 2002, dans une communication intitulée : «Les ambassadrices : des femmes dans un territoire d'hommes ». Publication des actes en cours.

${ }^{29}$ Textes reproduits dans Anne-Marie HOUDEBINE-GRAVAUD, La féminisation des noms de métiers, Paris, L'Harmattan, 1998, p. 197.

${ }^{30}$ Courriel de madame D., ambassadrice de 1991 à 1998, à l'auteur, 15 juillet 2001.

${ }^{31}$ Notamment dans des lettres adressées à l'auteur par madame Elisabeth Dahan, ministre plénipotentiaire, du 23 juillet 2001 ; de madame Bérengère Quincy, ambassadrice de France auprès de l'Office des Nations Unies et des
} 
Il est à noter que les six première femmes nommées ambassadeurs, étaient des célibataires. Cela reflète un aspect bien connu de l'accession des femmes à des postes à responsabilités : la disponibilité, l'engagement total dans le travail, etc. Pour les diplomates, la nomination de célibataires présentent en outre l'avantage d'éviter des problèmes de protocole. En effet, comment considère t-on, et comment appelle t-on tout simplement, un mari de femme ambassadeur et plus tard d'ambassadrice ? ${ }^{32}$ On conçoit que le terme de «Monsieur l'Ambassadeur » ne convienne pas, pas plus qu'une invitation adressée à "Madame et Monsieur l'Ambassadrice »... Certes ce genre de préoccupations protocolaires peut sembler dérisoire, mais les pratiques sont révélatrices des conceptions. Comment le Quai d'Orsay, par exemple, a t-il organisé le protocole à l'égard des femmes qui représentent leur pays en France $?^{33} \mathrm{Y}$ a t-il eu dans ce domaine une évolution parallèle à la très relative féminisation du corps diplomatique français?

Le nombre de femmes occupant des postes de chefs de mission n'a véritablement augmenté que dans les années 1990 et en particulier depuis quelques années. On comptait trois femmes ambassadeurs en 1982, 16 en $2002^{34}$. Et d'autres femmes représentent la France auprès des organisations internationales, mais nous y reviendrons. Avec l'évocation du nombre, la question de la parité apparaît. Il conviendrait de déterminer les différentes vagues de nominations de femmes à des postes d'ambassadrices, 1998 semblant être une année marquante dans ce domaine. Mais il faut évoquer également dans quels pays les femmes sont envoyées représenter la France.

En effet, à y regarder de plus près - et quelle que soit l'année de référence parmi les trente dernières - on constate que le nombre d'ambassades «féminines » est plus important que le nombre d'ambassadrices. Cela s'explique par le fait que certaines diplomates sont appelées à représenter la France auprès de plusieurs Etats voisins. Ainsi, en 1983, Mademoiselle Claude Lafontaine est l'ambassadrice de la France dans pas moins de huit Etats : Antigua et Barbuda, Barbade, Dominique, Grenade, Guyana, Saint-Vincent-et-lesGrenadines, Sainte-Lucie, Trinité et Tobago ! Dans les années 1980 et 1990, d'autres femmes occupent également des postes multiples auprès de petits Etats, essentiellement dans les Caraïbes, en Amérique centrale (Belize et Salvador), et en Afrique australe (Lesotho et Afrique du Sud, Mozambique et Swaziland). Quelle interprétation donnée à ces nominations multiples dans des Etats qui ne sont ni des grandes puissances, ni des pays avec lesquels la France entretient des relations privilégiées? Il faut noter que lorsque des hommes sont nommés dans ces régions, eux aussi doivent exercer plusieurs missions. Par ailleurs, depuis la fin des années 1990, cette pratique de confier plus particulièrement à des femmes des missions multiples semble avoir été abandonnée. Néanmoins, on remarque que les petits Etats ont toujours été et demeurent davantage susceptibles d'accueillir des femmes diplomates.

Il semble même que l'on peut déterminer des ensembles de pays davantage ouverts à la nomination des femmes. Dans les années 1970 et 1980, le Quai d'Orsay envoie des femmes pour représenter la France dans trois pays communistes : la RDA, la Bulgarie et la Hongrie. L'ouverture de postes diplomatiques français dans les Etats créés par la désintégration de l'URSS a permis à des femmes d'accéder depuis les années 1990 aux ambassades en Lettonie,

\footnotetext{
Organisations Internationales, du 5 septembre 2001 ; et de madame Marie-France Pagnier, ambassadrice de France à Sri Lanka et aux Maldives, du 14 septembre 2001.

${ }^{32}$ Problème soulevé par Anne-Marie HOUDEBINE-GRAVAUD, La féminisation des noms de métiers... op.cit ., p. 19.

${ }_{33}^{33}$ En 2001, 19 chefs de missions étrangères en France étaient des femmes.

${ }^{34}$ Le dépouillement de l'Annuaire Diplomatique est également irremplaçable, mais on trouve également des chiffres pour 1982 dans Annick DAVISSE, Les femmes dans la fonction publique. Rapport au ministre de la Fonction publique et des réformes administratives, Paris, La Documentation française, 1983, 190 p. Pour cerner l'évolution générale : Marcel PINET (dir.), Histoire de la fonction publique, tome III : le XIXème et le XXème siècles, Paris, Nouvelle Librairie de France, 1993, p.416.
} 
Géorgie, Moldavie, Arménie, Estonie et Azerbaïdjan. L'Asie du Sud et du Su-Est semble également être une région vers laquelle le Quai d'Orsay envoie plus volontiers les femmes : Bengladesh, Malaisie, Sri Lanka et Maldives, Philippines et Palau. Des recherches fines et précises pourraient permettre de confirmer ou d'infirmer des thèses qui peuvent être avancées. Le Quai d'Orsay n'a t-il pas cantonné les femmes dans des représentations difficiles et de moindre importance? Ou dans celles qui nécessitaient des compétences linguistiques très particulières ? Cette question étant à rapprocher de la plus grande proportion de femmes dans le cadre d'Orient déjà évoquée...

Toute nomination diplomatique est interactive. Ainsi, on peut penser que c'est parce les pays du Nord de l'Europe ont été et demeurent les plus en avance sur la question de la parité hommes/femmes, que la France leur envoie plus volontiers des femmes pour la représenter (Norvège dans les années 1980, Suède dans les années 1990, Pays-Bas aujourd'hui). Les récentes nominations d'ambassadrices de France à Barhein et en Azerbaïdjan ont-elles été favorisées par le fait que ces deux pays soient représentées en France par des femmes? A l'inverse, le Quai d'Orsay n'envisage pas la nomination d'ambassadrices auprès de pays dont la culture et la religion empêcheraient l'agrément d'une femme diplomate et auprès desquels une simple proposition de cette nature serait considérée comme une sorte provocation. Cette impossibilité n'est-elle pas rappelée lorsque l'on veut justifier l'absence de parité hommes/femmes dans les ambassades ? Mais comment comprendre les motivations de la diplomatie française envoyant une ambassadrice en Libye... Bien entendu, il ne s'agit de rechercher d'éventuels caractères féminins aux missions remplies par les femmes. "Les diplomates fournissent aux responsables politiques des éléments d'information et d'analyse et sont des agents d'exécution », écrit une ambassadrice à la retraite, qui «ne croi[t] pas plus à une spécificité féminine en la matière qu'à une homogénéité des comportements masculins $» 35$.

Cependant les affectations des femmes diplomates semblent bien obéir à des règles implicites. On constate par exemple qu'aucune femme n'a jamais représenté la France auprès de l'ONU, de l'OTAN ou du Conseil de l'Atlantique Nord depuis leur création. Les affaires internationales et militaires semblent donc réservées de facto aux hommes. Les affaires économiques sont désormais ouvertes aux femmes, avec deux représentantes françaises auprès de l'OCDE depuis 1993. Depuis 1981 pas moins de quatre femmes ont représenté la France auprès de l'UNESCO. Or, ce poste est basé à Paris - tout comme celui de l'OCDE - et traite de questions considérées comme moins capitales que celles citées plus haut. De plus, ce ne sont pas des diplomates qui ont occupé ces postes, mais plutôt des politiques ${ }^{36}$. On comprend donc qu'il faut prendre en compte un nombre important de variables pour parvenir à avancer des explications.

\section{La nécessité du recours aux témoignages}

Pour pouvoir traiter toutes ces questions et d'autres, quelles sont les sources à la disposition des chercheurs qui s'intéressent à la place des femmes dans la politique étrangère de la France ? L'Annuaire Diplomatique et Consulaire de la République Française, édité chaque année par l'Imprimerie Nationale, constitue une mine de renseignements très riche. On y trouve en particulier les résultats des «concours et examens pour le recrutement des agents diplomatiques et consulaires ». Les «listes d'ancienneté » permettent de calculer la proportion de femmes dans chaque catégorie de personnel. Les «états de service du personnel » sont eux très utiles pour retracer les carrières des femmes diplomates et les comparer à celles des hommes. Les nomenclatures chronologiques et annuelles des

\footnotetext{
${ }^{35}$ Lettre de madame R. directeur au Quai d'Orsay dans les années 1980-1990, à l'auteur, 30 juillet 2001.

${ }^{36}$ Il s'agit de mesdames Baudrier, Halimi, Cabana et De Panafieu.
} 
« représentations diplomatiques et consulaires françaises à l'étranger et auprès des organisations, commissions et conférences internationales ${ }^{37}$ fournissent également des renseignements sur l'affectation des femmes dans des postes à l'étranger.

L'utilisation des archives du ministère des Affaires étrangères est beaucoup plus délicate. En ce qui concerne la féminisation du personnel du Quai d'Orsay, les textes et circulaires organisant le recrutement doivent être systématiquement dépouillées afin de voir si à une époque ou à une autre, des dispositions particulières ont été appliquées aux femmes. En dehors de ces documents, peu de sources sont susceptibles de fournir des renseignements notoires. Etant donné le caractère très récent de la période étudiée, on comprend que la série «personnel » des archives du Quai d'Orsay ne soit pas encore communicable. De plus, les raisons de la nomination de telle femme - ou de tel homme d'ailleurs - à un poste d'ambassadeur sont souvent obscures, car résultant de beaucoup d'éléments : mouvement diplomatique, prise en compte des carrières, motivations politiques, nécessités internationales, influence de réseaux, etc. Rappelons en outre que «les affectations aux emplois de chef de mission diplomatique sont prononcées par décret du Président de la République pris en conseil des ministres ${ }^{38}$. Si ces nominations laissent des traces dans les publications officielles (Journal Officiel), leurs motivations ne sont pas explicitées.

On l'aura compris en lisant les lignes qui précèdent, le recours aux témoignages des acteurs et des actrices de la vie politique et diplomatique de la France est donc indispensable afin de palier à la pauvreté et à la difficulté d'accès aux archives. Les deux ouvrages de souvenirs laissés par Suzanne Bidault, et déjà évoqués, n’ont pas été étudiés en tant que source permettant de mieux cerner les premiers pas d'une femme conseillère des Affaires étrangères. Il y a là matière à une étude biographique et critique. En effet, c'est surtout Giraudoux qui est cité lorsqu'on évoque le parcours de Suzanne Borel. Les nombreux témoignages laissés par les diplomates français du XXème siècle évoquent bien les ambassadrices, mais ce sont des épouses des ambassadeurs dont il s'agit ${ }^{39}$. La dernière livraison en date de ces souvenirs de diplomates est l'ouvrage de l'ambassadeur Bernard Dorin. Le diplomate pointe d'une manière très pertinente les nécessaires adaptations du Quai d'Orsay au monde d'aujourd'hui... mais n'évoque pas la féminisation du corps diplomatique. En revanche, il propose qu'une petite décoration vienne récompenser les épouses des ambassadeurs, qui effectuent «bénévolement les obligations sociales» dans les ambassades ! ${ }^{40}$ Andrée Dore-Audibert, dont la vie a été dédiée à l'action sociale et à l'Afrique, demande bien autre chose dans ses souvenirs d'épouse d'ambassadeur ${ }^{41}$. Elle souhaite l'instauration d'un véritable statut pour les épouses de diplomates. En féministe, elle dénonce l'absence de parité au sein du corps diplomatique français.

Il faut regretter que la plume des ambassadrices ne livre pas d'indications sur les femmes dans la politique étrangère de la France, puisqu'aucune d'entre elles n'a rédigé de souvenirs. Une ambassadrice précise : «lors de mon admission à la retraite à l'aube du XXIème siècle, j'ai décidé de ne plus faire de travaux écrits, et de ne plus prendre la parole en public ». Et d'évoquer un métier de diplomate «qui impose une remise en question

\footnotetext{
${ }^{37}$ Les appellations des titres et chapitres citées ici sont celles de l'édition 2002 de L'Annuaire Diplomatique.

${ }^{38}$ Article 66 du décret $n^{\circ} 69-222$ du 6 mars 1969 relatif au statut particulier des agents diplomatiques et consulaires.

${ }^{39} \mathrm{Au}$ milieu de beaucoup d'autres : Jean-Baptiste BARBIER, Un frac de Nessus, Rome, L'Alveare, 1951 ; Jacques BAEYENS, Au bout du Quai, Paris, Fayard, 1975 ; et plus récemment Bernard DESTREMEAU, Quai d'Orsay, derrière la façade, Paris, Plon, 1994, p.42 à 45.

${ }^{40}$ Bernard DORIN, Appelez-moi Excellence, Paris, Stanke, 2000, p.63.

${ }^{41}$ Andrée DORE-AUDIBERT, Propos irrévérencieux d'une épouse d'ambassadeur, Paris, Karthala, 2002, 228 pages. L'auteure est l'épouse de l'ambassadeur Jean Audibert $(\dagger)$ en poste à Vienne, Bruxelles et Alger de 1984 à 1992.
} 
personnelle fréquente, ce qui devrait inciter à l'humilité ${ }^{42}$. Néanmoins, les témoignages des principales actrices doivent donc être suscités. Il est juste temps d'entreprendre un véritable travail d'histoire orale afin de recueillir une mémoire vive absolument nécessaire à l'historien du temps présent. On ne peut qu'encourager des travaux d'enregistrement systématique des expériences des femmes diplomates. Mais il faut avant tout convaincre les principales intéressées de l'importance de cette démarche. A cet égard, le témoignage de madame Elisabeth Dahan, lors d'une journée d'études consacrée aux femmes dans la politique étrangère de la France au XX ème siècle : études et témoignages, a constitué une première qui peut ouvrir la voie à d'autres ${ }^{43}$. En effet, cette histoire ne se fera que très difficilement si les actrices elles-mêmes - et les acteurs - ne livrent pas leurs expériences, leurs sentiments et leurs analyses sur les thèmes de recherche des universitaires. Le chantier est donc ouvert. Les perspectives et les travaux à entreprendre sont nombreux pour parvenir à une meilleure connaissance de la politique étrangère de la France contemporaine.

Yves Denéchère

Maître de conférences en histoire contemporaine Université d'Angers

\footnotetext{
${ }^{42}$ Lettre de madame D., ambassadrice de 1996 à 1999, à l'auteur, 31 juillet 2001.

${ }^{43}$ Journée d'études de l'HIRES (centre d'histoire des régulations et politiques sociales) organisée par Yves Denéchère le 21 septembre 2002 à l'Université d'Angers. On a pu y entendre les témoignages de Françoise Gaspard, Andrée Dore-Audibert et Elisabeth Dahan. Madame Dahan est ministre plénipotentiaire, conseiller pour les relations internationales auprès du Président du Conseil économique et social. Elle a été ambassadrice à Sri Lanka et aux Maldives de 1996 à 2000. Les actes de cette journée d'études feront l'objet d'une publication.
} 